\title{
Scoping review of the morphology and anthropometry of Tessier craniofacial clefts numbers 3 and 4
}

\author{
Abiola Omodan ${ }^{* *}$ D, Pamela Pillay ${ }^{1}$, Lelika Lazarus ${ }^{1}$, Anil $_{\text {Madaree }}^{2}$ and Kapil Satyapal ${ }^{1}$
}

\begin{abstract}
Background: In 2016, WHO reported a death rate of 303,000 newborns before 4 weeks of age due to congenital anomalies. Those that survive congenital anomalies may have long-term disabilities which may have significant impacts on the individual, their families, the healthcare system, and societies. Tessier craniofacial clefts numbers 3 and 4 are congenital anomalies that result in a partial or total defect of craniofacial tissues thereby seriously influencing the patient's appearance and impair normal functioning. Therefore, understanding these defects is paramount to relieving the burden caused by this disability. The objective of this review was to examine the literature on the understanding of the knowledge of morphology and anthropometry of Tessier craniofacial clefts numbers 3 and 4 so that areas yet to be fully understood by research can be mapped out for future research.
\end{abstract}

Methods and analysis: A scoping review for literature on patients who have Tessier craniofacial clefts numbers 3 and 4 was conducted. Relevant studies from 1976 to the present were identified. The following databases were searched for peer-reviewed literature viz., PubMed, MEDLINE, EBSCOhost, Google Scholar, and the Cochrane library. The study selection was guided by the eligibility criteria. A data table was designed to extract information from the literature. The result of this study was reported using the Preferred Reporting Items for Systematic reviews and Meta-analyses (PRISMA). The quality of the included studies was assessed using the Mixed Method Appraisal Tool (MMAT).

Result: Thirty-three studies met the inclusion criteria. The majority of the studies included were conducted in middle-income countries (54.5\%) and some in high-income countries (45.5\%); none was recorded from lowincome countries. The total available sample size from the studies was 120 with a dominant male population of 67 (55.8\%) and female 53 (44.2\%). The majority (97\%) of the studies reported on the knowledge of morphology while $12.1 \%$ of the included studies reported on anthropometry. Of the 33 included studies, 32 scored the highest quality (76-100\%) from the quality assessment.

Discussion: The findings from this review show evidence of the knowledge of morphology and the knowledge of anthropometry of Tessier craniofacial clefts numbers 3 and 4 . However, these knowledges have not translated to universally recognized ways of repairing and documenting these clefts due to the sparse amount of studies on Tessier craniofacial clefts numbers 3 and 4.

Keywords: Craniofacial clefts, Tessier number 3, Tessier number 4, Morphology, Anthropometry

\footnotetext{
* Correspondence: 216077058@stu.ukzn.ac.za

'Department of Clinical Anatomy, School of Laboratory Medicine and

Medical Sciences, University of KwaZulu-Natal, Durban, South Africa

Full list of author information is available at the end of the article
}

(c) The Author(s). 2019 Open Access This article is distributed under the terms of the Creative Commons Attribution 4.0 International License (http://creativecommons.org/licenses/by/4.0/), which permits unrestricted use, distribution, and reproduction in any medium, provided you give appropriate credit to the original author(s) and the source, provide a link to the Creative Commons license, and indicate if changes were made. The Creative Commons Public Domain Dedication waiver (http://creativecommons.org/publicdomain/zero/1.0/) applies to the data made available in this article, unless otherwise stated. 


\section{Background}

Congenital craniofacial cleft deformity is a partial or total defect of the soft tissue, bone, or a combination of these tissues in the craniofacial region. The severity of this deformity can range from relatively minor skin and soft tissue deformities to major bony abnormalities or defects of the cranial, orbital, or facial skeleton [1]. Craniofacial clefts can occur anywhere in the craniofacial area and may seriously influence the patient's appearance and impair normal functioning [1]. The incidence of the rare facial clefts is between 1.43 and 4.85 per 100,000 births [2]. Craniofacial anomalies affect a significant proportion of society with the ratio varying considerably by geographical area and ethnic grouping [3]. The costs of these anomalies in terms of morbidity, healthcare, emotional disturbance, and social and workplace exclusion are considerable for affected individuals, their families, and society [3].

According to the World Health Organization (WHO), 303,000 newborns die within the first 4 weeks per year worldwide from congenital anomalies, of which Tessier craniofacial clefts numbers 3 and 4 are included [4]. Since one of the Sustainable Development Goals (SDG) of the World Health Organization (WHO), as well as South Africa, is to ensure healthy lives and promotion of well-being at all ages, knowledge and understanding how to solve this problem are critical for these anomalies [5].

Rare craniofacial clefts pose the most significant reconstructive challenge to the craniofacial surgeon today because of their variability and complexity [6]. Facial cleft surgery publications are sparse due to the rarity of the disorders, and consensus has yet to develop on standardized landmarks, reference measurements, and principles of repair [7]. Understanding the soft tissue and skeletal deformity is basic to any reconstructive surgical procedure of the face [2]. There is little evidence in the literature to suggest that much is being done to address this issue.

Therefore, the objective of this review is to map out evidence of the knowledge of morphology (particular forms in which these clefts present) and anthropometry (description of these clefts using measurements or the use of some form of measurements in their repair) of Tessier craniofacial clefts numbers 3 and 4. Researchers in the field of craniofacial anomalies may benefit from the findings of this review as it highlights areas still undiscovered in the pursuit of understanding the variant anatomy of Tessier craniofacial clefts numbers 3 and 4 .

\section{Methods}

This study on the knowledge of morphology and the knowledge of anthropometry of Tessier craniofacial clefts numbers 3 and 4 is a part of a larger study looking at "Understanding Tessier craniofacial clefts numbers 3 and 4: A scoping review," which is a part of a study looking at the "Anatomical classification of Tessier craniofacial clefts numbers 3 and 4 in a select South African population."

A scoping review research method is defined as a "type of research synthesis that aims to map the literature on a particular topic and provide opportunity to identify gaps to guide future studies." [8].

This scoping review began with the establishment of a research team consisting of individuals with expertise in epidemiology and research synthesis [9]. The team advised on the broad research question to be addressed and the overall study protocol, including identification of search terms and selection of databases to search. The methodology for this scoping review was based on the framework outlined by Arksey and O'Malley (2005) and ensuing recommendations made by Levac and colleagues (2010) [9, 10]. The review included the following five key phases: (1) identifying the research question; (2) identifying relevant studies; (3) study selection; (4) charting the data; and (5) collating, summarizing, and reporting the results. A detailed review protocol can be obtained from the primary author upon request.

\section{Research question}

This review was guided by the main question "What types of research on Tessier craniofacial clefts number 3 and 4 have been reported?" and a sub question "In which countries (High, Middle OR low income) are research on Tessier craniofacial clefts number 3 and 4 being reported?". We applied the PCC (Population, Concept and Context) framework to determine the eligibility and appropriateness of the primary research question. The results of the scoping review were reported using the PRISMA (Preferred Reporting Items for Systematic reviews and Meta-analysis) guidelines. See Additional file 1.

\section{Data sources and search strategies}

The initial search was implemented on 29 November 2017, in four electronic databases: viz., Google Scholar, EBSCOhost (Academic search complete, Educational source, Health source, Nursing/Academic, Medline with full text, Medline), PubMed, and the Cochrane library. The databases were selected to be comprehensive. No limits were made on language; however, there was a date limit (1976 and above, which is the year that the current accepted classification by Tessier was published). The search query consisted of terms considered by the authors to describe the scoping review and its methodology: Tessier clefts, Tessier number 3, Tessier number 4 , Tessier number 3 and number 4 morphology, Treatment, Treatment outcome, and anthropometry. The 
search query was tailored to the specific requirements of each database. The entire literature search strategy, reflecting dates, database, search terms, and the results were documented.

\section{Citation management}

All citations were imported into EndNote X7, and duplicate citations were removed manually with further duplicates removed when found later in the process before they were subsequently used in title and abstract screening and data characterization of full articles.

\section{Eligibility criteria}

A set of questions was used to assess the relevance of studies identified in the search. Studies were eligible for inclusion if they reported on Tessier craniofacial clefts number 3 or number 4, if they were done in 1976 and later, and if the studies had information on the morphology and anthropometry of Tessier craniofacial clefts numbers 3 or 4 . Studies that were not primarily on Tessier craniofacial clefts number 3 or number 4 were excluded as well as studies done pre-1976.

\section{Title, abstract, and full-article relevance screening}

For the first level of screening, one member of the team screened the titles from the databases and exported eligible articles to an Endnote library ready for abstract screening. For the second level, an abstract relevance screening form was developed by the authors and reviewed by the research team. Two members of the research team (AO and DK) independently screened the abstracts. Any disagreements at this level of the research necessitated both reviewers to meet for discussion until a common consensus was reached. All citations deemed relevant after title and abstract screening were procured for subsequent review of the full-text article.

For articles that could not be obtained through institutional holdings available to the authors, attempts were made to contact the source author or journal for assistance in procuring the article. The third level of screening involved creating a full-article screening form, and two members of the team (AO and TS) independently screen the full articles. Disagreements at this stage were however resolved by involving a third reviewer (TMT). The degree of agreement for the full-article screening was calculated with the overall kappa being 0.989, where a kappa of greater than 0.8 is considered to represent a high level of agreement [11] (see Additional file 1). Reviewers met throughout the screening process to resolve conflicts and discuss any uncertainties related to study selection [9].

\section{Quality assessment of individual studies}

All the 33 included primary studies underwent methodological quality assessment using the Mixed Methods Appraisal Tool (MMAT)-version 2011 [12] (see Additional file 2). A form was designed by the authors, and it was then piloted by the research team before it was used on the articles. The studies were assessed in the following domains: the clarity of the research question, relevance of the sampling strategy to the research question, appropriateness of the measurements, and appropriate representation of the population under study. An overall quality percentage score for each of the included studies was calculated, and the scores were interpreted as low quality $(\leq 50 \%)$, average quality $(51-75 \%)$, and high quality (76-100\%).

\section{Data characterization}

A form was developed by the authors to confirm relevance and to extract study characteristics such as author and date, title, main objective, knowledge of morphology, knowledge of treatment, knowledge of treatment outcome, knowledge of clinical spectrum, knowledge of anthropometry, most significant outcome, study design, other significant findings, country of the study, and high- or middle/low-income country. Other information such as the percentage of male or female and age range of the population was extracted. The research team reviewed this form and slight modifications were made before use.

\section{Data summary and analysis}

The data were compiled in a single spreadsheet and imported into Microsoft Excel 2010. Content analysis of each emerging theme was done. The emerging themes identified for this paper included the knowledge of anthropometry and the knowledge of morphology. These themes were extracted from all studies that were included.

\section{Patient and public involvement}

Patients were not involved in this study.

\section{Results}

The original search conducted in November 2017 yielded 5529 potentially relevant citations. After deduplication and relevance screening, 44 citations met the eligibility criteria based on the title and abstract and the corresponding full-text articles were procured for review. After data characterization of the full articles, 33 studies remained and they were included in the analysis (see Additional file 2). The flow of articles through identification to final inclusion is represented in Fig. 1.

Of the 11 articles excluded at full-article screening, five articles could not be procured and thus were not 


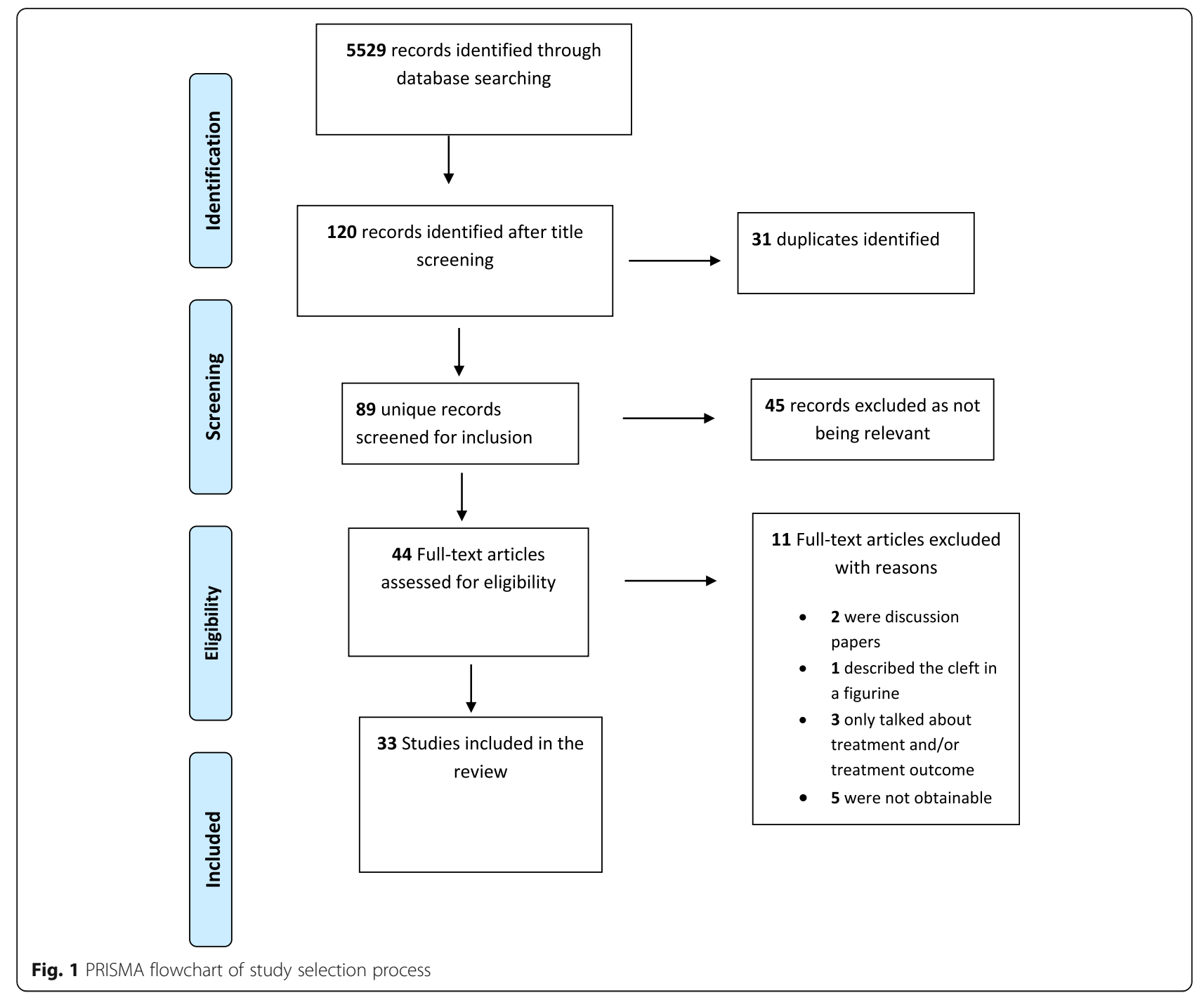

included in the review [13-17]. Two articles were discussion papers $[18,19]$. Bartlett discussed the study of [6] which is one of the included studies, while Resnick and Kawamoto discussed the morphology and treatment. Three articles were not relevant as they did not illustrate evidence of the knowledge of anthropometry nor the knowledge of morphology which are the themes for this paper [20-22]. Aleman and Martinez described the cleft in an ancient figurine [23].

\section{Characteristics of included studies}

Table 1 shows the characteristics of the included studies while Fig. 2 shows a graphical representation of the advent of the knowledge of morphology and anthropometry in literature pertaining to Tessier craniofacial clefts number 3 and number 4 . The majority (54.5\%) of the included studies were done in middle-income countries [24-41], 45.5\% in high-income countries [6, 7, 42-54], and none was carried out in low-income countries. All included studies were published between 1980 and 2017 . The total available sample size from the studies was 120 with a dominant male population of $67(55.8 \%)$ and female $53(44.2 \%)$. All the included studies were either case reports or case series. The majority (97\%) of the studies reported on the knowledge of morphology [7, 24-54] while $12.1 \%$ of the included studies reported on anthropometry $[6,7,40,45]$.

\section{Quality of evidence from included primary studies}

All the 33 primary studies underwent methodological quality assessment using the Mixed Methods Appraisal Tool (MMAT)-version 2011 [12]. The studies were assessed based on all the categorized domains. Of the 33 included studies, 32 scored the highest (76-100\%) [2451, 53, 54]. The last one was not assessed further after scoring a "No" in the first question determining if there was a clear research question [52]. 


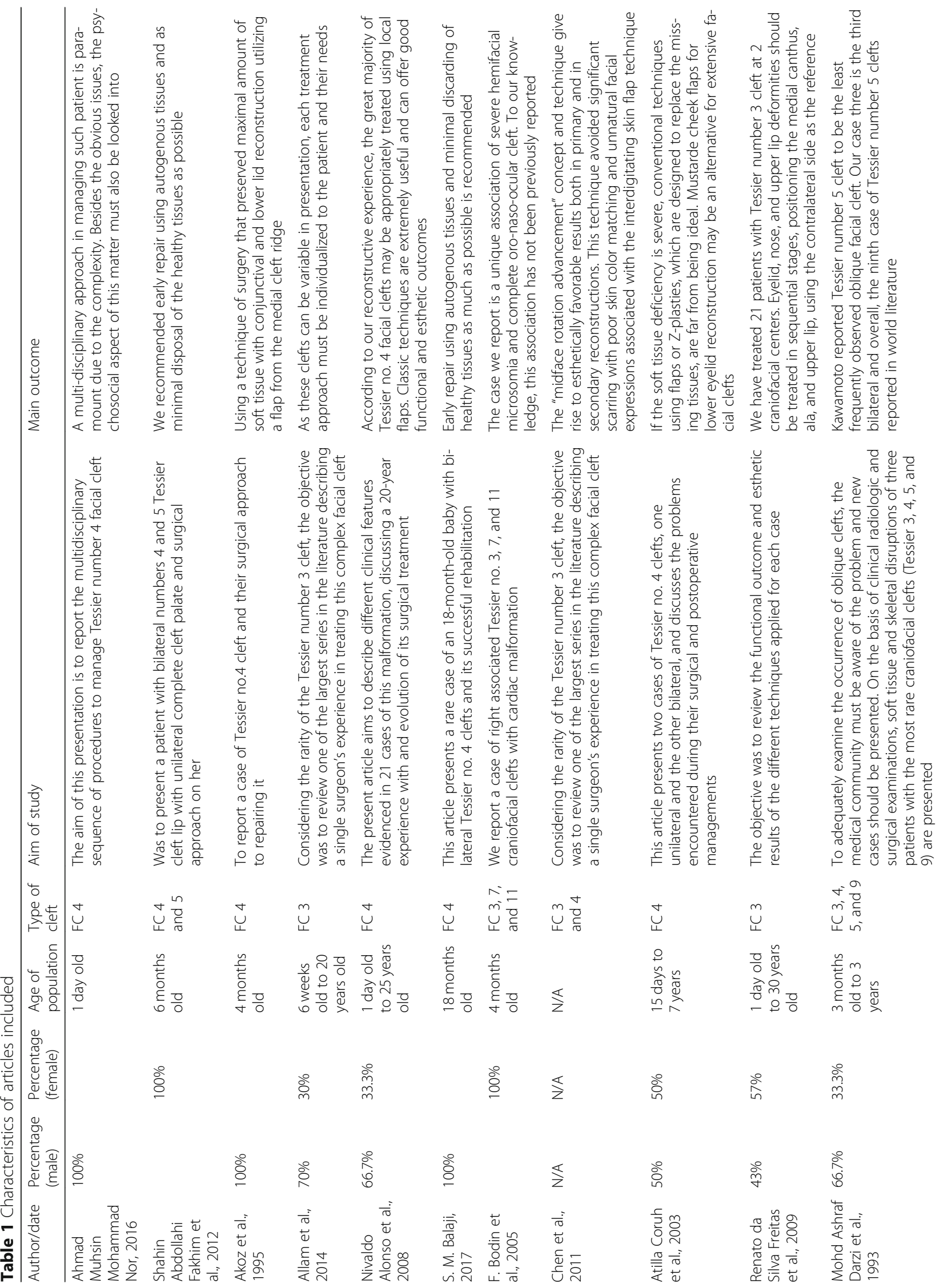




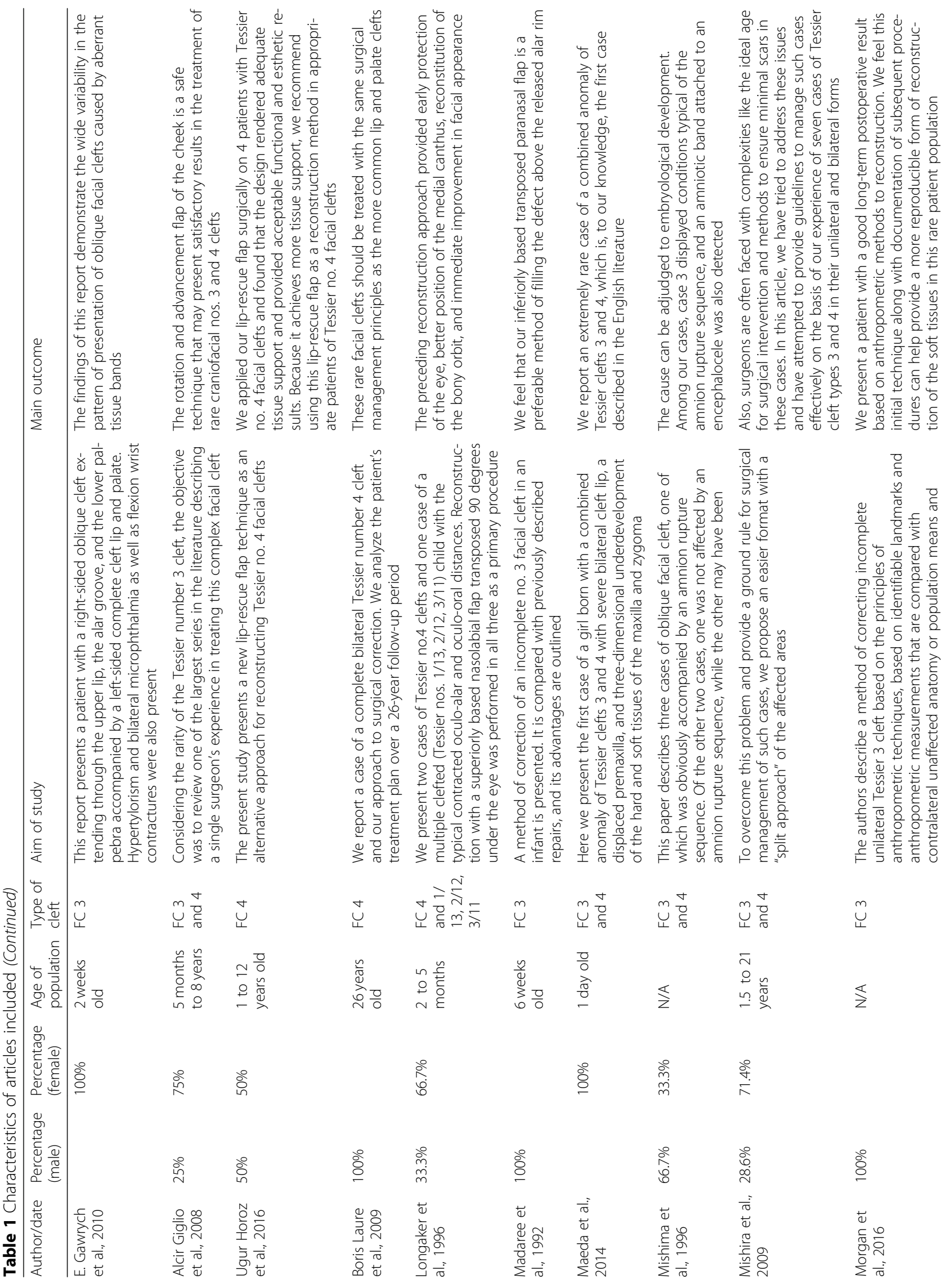




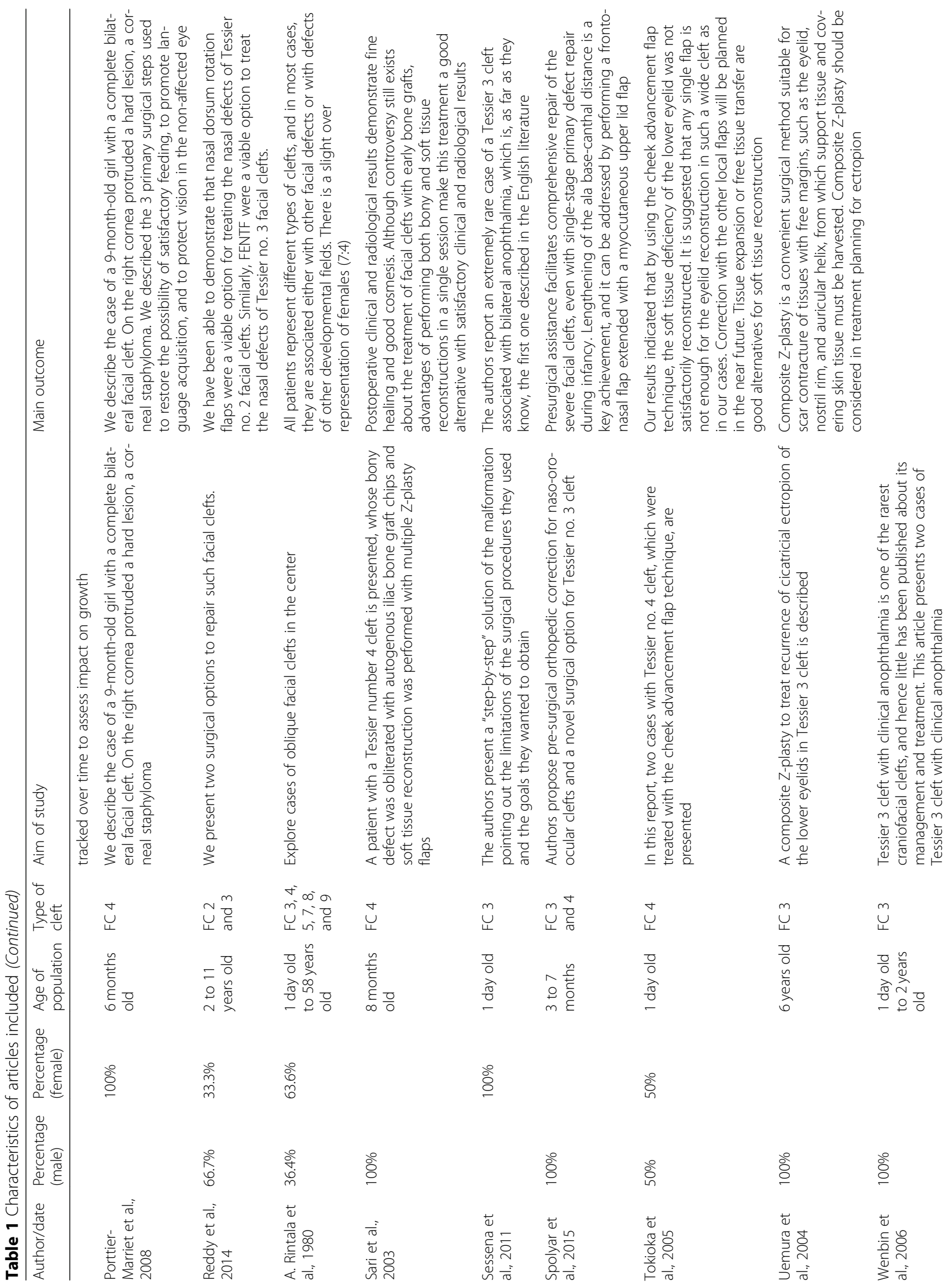




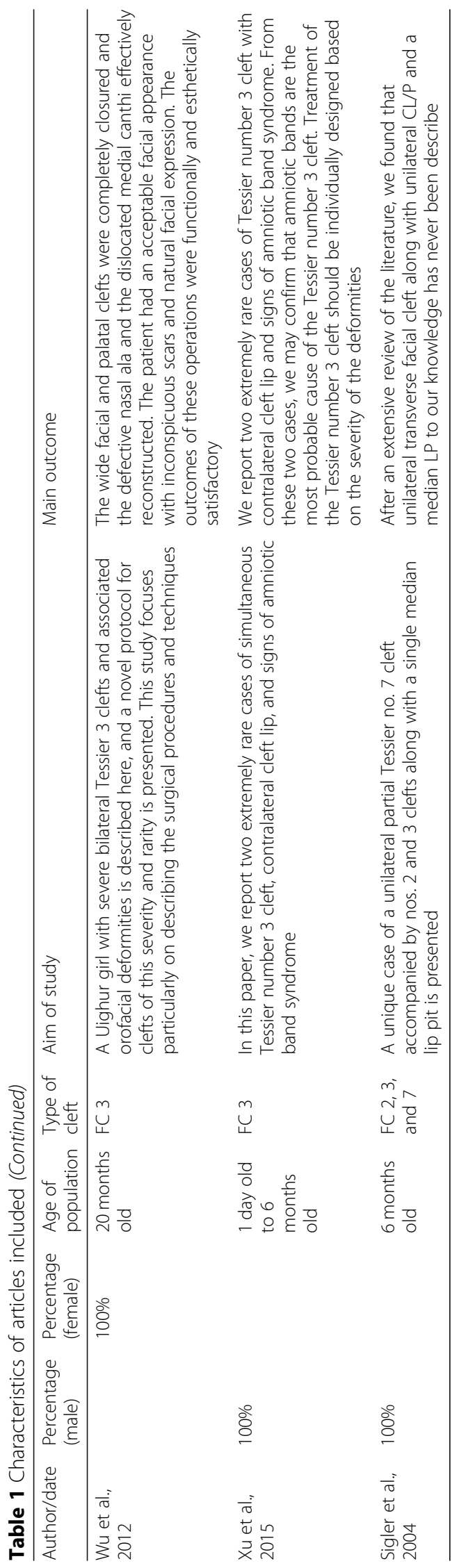




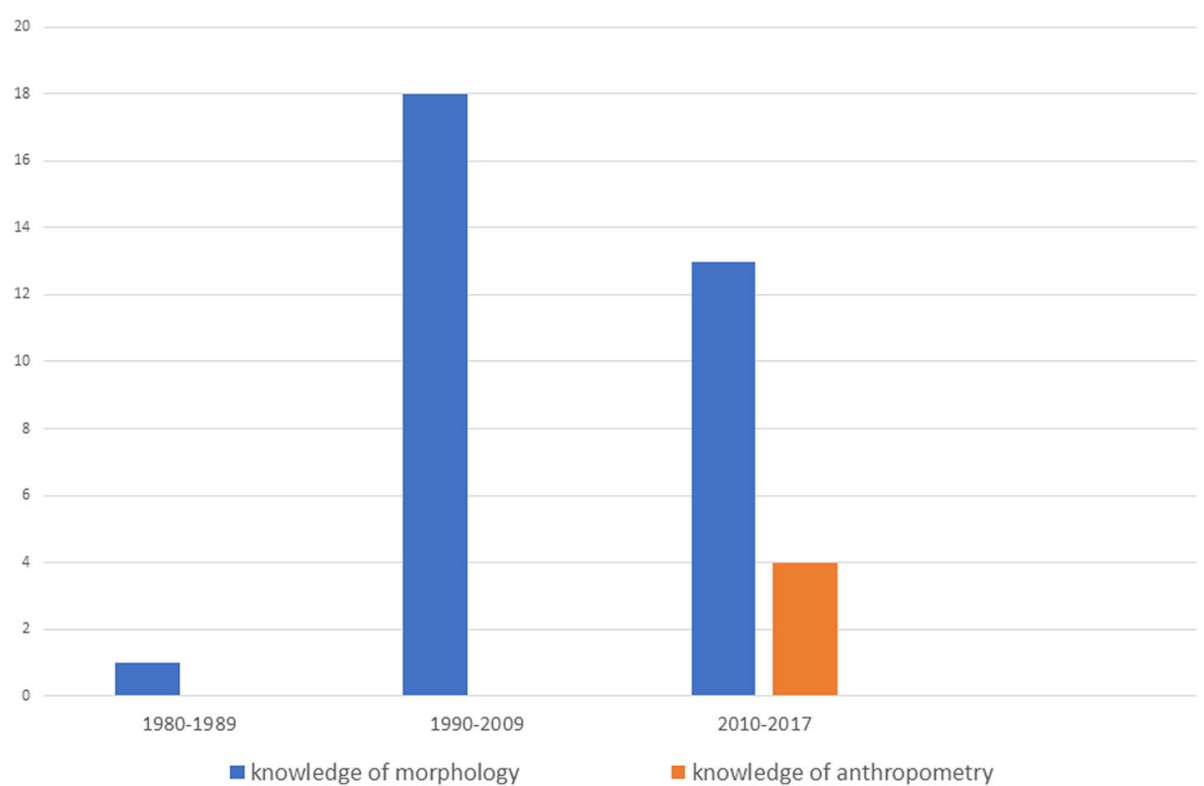

Fig. 2 Graph showing timeline of advent of knowledge of morphometry and anthropometry in the literature in the past 3 decades. $X$-axis displays the year and $y$-axis illustrates number of articles

\section{Themes from included studies \\ Knowledge of anthropometry of Tessier craniofacial clefts number 3 and number 4}

Four of the $33(12.1 \%)$ included studies showed varying levels of the knowledge of anthropometry in discussing Tessier craniofacial clefts number 3 and number 4. Two of these studies showed the knowledge of anthropometry in the surgical reconstruction of the defects $[6,7]$ while the other two displayed the knowledge of anthropometry in defining the clefts and then reconstruction [40, 45].

(a) Knowledge of anthropometry in cleft definition and reconstruction

Two of the included studies showed evidence of the knowledge of anthropometry not only in the reconstruction of the defects but also initially in defining the clefts [40, 45]. One of the studies was done in a high-income country (USA) and the other in a middle-income country (China). In their study, $\mathrm{Wu}$ et al. aimed at describing a novel protocol for these clefts and attributed the satisfactory functional and esthetic outcomes to the novelty of the procedure [40]. These studies show a lack of literature on the emerging technique of anthropometry in the correction of defects of Tessier craniofacial clefts numbers 3 and 4 .

(b) Knowledge of anthropometry in surgical reconstruction

Two studies showed the knowledge of anthropometry during surgical reconstruction of the defects in Tessier craniofacial clefts number 3 and number 4 [6, 7]. Both studies were carried out in high-income countries (Taiwan, USA). In a study by Chen and colleagues aimed at reviewing one of the largest series in the literature describing a single surgeon's experience in treating this complex facial cleft, findings showed that previous treatment options paid little attention to the anatomical repair of the affected facial musculature which has led to sub-optimal results with conspicuous facial scars, poor color matching of the cheek and nasal flaps, and unnatural facial expression [6]. The study by Morgan et al. described a method of correcting incomplete unilateral Tessier 3 cleft based on the principles of anthropometric techniques showing that consensus has yet to be developed on standardized landmarks, reference measurements, and principles of repair due to sparse publications [7]. Evidence from these studies documented that knowledge of anthropometry is key to a satisfactory outcome in the surgical management of these clefts and there is a scarcity of publication on this knowledge, and this has made arriving at a consensus on standardized landmarks, reference measurements, and principle of repair difficult thus far.

\section{Knowledge of morphology}

Thirty-two (97\%) of the included studies showed evidence of the knowledge of morphology of Tessier craniofacial clefts numbers 3 and 4 [7, 24-54]. Evidence shows that the knowledge of morphology was expressed by describing the clefts as complete, while five of the 
studies (15.6\%) described a form of the cleft as incomplete $[26,27,33,34,41]$.

(a) Knowledge of morphology of complete clefts

Thirty-one (96.9\%) of the studies that showed the knowledge of morphology showed evidence of knowledge of a complete cleft [7, 24-32, 34-54]. Alonso et al., in their study which was on the different clinical features in 21 cases of number 4 cleft, described the complete cleft as consisting of a cleft lip, lateral to the Cupid's bow, which crosses superiorly up to the lower eyelid, decreasing the oro-ocular distance [28]. While Allam et al., in a study reviewing one of the largest series of number 3 clefts in the literature, described the complete number 3 cleft as extending from the philtrum of the lip to the medial canthus of the eye with the foreshortening of this distance with affectation of the nasal ala [27]. Evidence from these studies show that there is a generally acceptable knowledge of the morphology of a complete cleft of Tessier craniofacial clefts numbers 3 and 4 .

\section{(b) Knowledge of morphology of incomplete clefts}

Five $(12.1 \%)$ of the 32 studies that reported on the evidence of the knowledge of morphology showed evidence of incomplete cleft $[26,27,33,34,41]$. In their studies, Giglio and colleagues who aimed at reviewing a large collection of the rare cleft number based on a single surgeon's perspective and Madaree et al., whose aim was to describe a method of correction of an incomplete number 3 cleft while comparing with previously documented methods, described the incomplete cleft as sparing the lip [33, 34]. Evidence from these studies show that there is a paucity of literature on the morphology of the incomplete Tessier craniofacial clefts numbers 3 and 4 .

\section{Discussion}

This study sets out to map out evidence of the knowledge of morphology and the knowledge of anthropometry of Tessier craniofacial clefts numbers 3 and 4 . A total of 33 unique articles were found that included the evidence of morphology and anthropometry of Tessier craniofacial clefts numbers 3 and 4 . The result of this study shows us that there have been studies on Tessier craniofacial clefts numbers 3 and 4 as far back as 1980 [45]; however, evidence of the knowledge of anthropometry began in the year 2011. In addition, evidence from these studies indicate that the knowledge of anthropometry is key to a satisfactory outcome in the surgical management of these clefts; however, there is a scarcity of publication on this knowledge and this has made arriving at a consensus on standardized landmarks, reference measurements, and principles of repair difficult thus far. Also, evidence from these studies show that there is a generally acceptable knowledge of the morphology of a complete cleft of Tessier craniofacial clefts numbers 3 and 4; however, there is a sparsity of literature on the morphology of the incomplete numbers 3 and 4 Tessier craniofacial clefts. Although the problem of facial clefts is a global issue [4], this study further revealed that studies on the knowledge of anthropometry of Tessier craniofacial clefts numbers 3 and 4 were mostly done in middle-income countries (54.5\%) while $45.5 \%$ was carried out in high-income countries, and none were done in low-income countries.

Cizmeci and Kuvat aimed at presenting a treatment option for these rare clefts and also reiterated that little is being published about the treatment and management of these clefts due to its rarity [20].

To the best of our knowledge, this is the first scoping review to map evidence on the knowledge of morphology and the knowledge of anthropometry in Tessier craniofacial clefts numbers 3 and 4 . An extensive search strategy, which helped in the identification of a considerable number of studies, was conducted in this study. The study followed clear screening processes using keywords, which were guided by study PCC nomenclature. A thorough data search using Boolean terms was conducted during the literature search to increase the chances of finding eligible studies for inclusion in this review. The degree of agreement between the reviewers was significant $(>0.05)$ after the full-article screening. The review also included a transparent methodological quality assessment of the included primary studies using the recommended MMAT tool [12].

Despite the reported strength of our study, the limitation we encountered was primarily the inability to retrieve some articles which might have been of benefit to the study [13-17]. This was despite efforts including but not limited to personal letters to the authors. Also, Chen $\mathrm{PK}-\mathrm{T}$, et al. did not distinguish male from the female participants in their study [6].

Our review has shown that there is little in the form of research publications on the morphology (especially incomplete clefts) and more importantly on the anthropometry of Tessier craniofacial clefts numbers 3 and 4 . We recommend to researchers that not only should more be done in documentation of incomplete clefts but more importantly research should be redirected mostly towards the emerging technique of anthropometry in the understanding and possibly finding a standardized way of managing the rare craniofacial clefts numbers 3 and 4. Also, we recommend that these studies require to be reported in low-resource countries as currently there is no evidence of such studies from these areas. 
To surgeons in the management of these complex rare facial clefts, we recommend the use of anthropometric techniques in the way the repairs are carried out as this will prove to be a more reproducible method of repair and will further contribute to having a standardized way of carrying out these complex yet rewarding surgeries.

\section{Conclusion}

Our findings suggest that the knowledge of morphology and the knowledge of anthropometry of Tessier craniofacial clefts numbers 3 and 4 exist albeit not fully harnessed. Furthermore, our review highlights the fact that no studies on these clefts are being done in low-income countries despite the global prevalence of this disease. This review also highlights the fact that knowledge of anthropometry is an emerging technique of solving the problem posed to surgeons of not having a standardized way of treating these defects. Further, studies should be encouraged in areas of anthropometry of Tessier craniofacial clefts numbers 3 and 4 as well as other aspects that affect the rare clefts Tessier numbers 3 and 4 such as their treatment, the outcome of this treatment, and possibly the clinical spectrum of their presentation.

\section{Additional files}

Additional file 1: PRISMA checklist. (DOC $64 \mathrm{~kb}$ )

Additional file 2: Calculation of degree of agreement for full-article screening between the two reviewers. (PDF $86 \mathrm{~kb}$ )

\section{Abbreviations}

MMAT: Mixed Method Appraisal Tool; PCC: Population, Concept, Context: PRISMA: Preferred Reporting Items for Systematic reviews and Meta-analyses; SDG: Sustainable Development Goals; USA: United States of America; WHO: World Health Organization

\section{Acknowledgements}

Special thanks to the College of Health Sciences and Library services at the University of KwaZulu-Natal for providing resources to help us with setting up and conducting this research study. Systematic review unit of the College of Health Sciences. DK and TS, and TMT.

\section{Funding}

This study was funded by the University of KwaZulu-Natal, College of Health Sciences Research Scholarship.

\section{Availability of data and materials}

All data generated or analyzed during this study are included in this published article [and its supplementary information files].

\section{Authors' contributions}

$\mathrm{AO}$ and PP conceptualized and designed the study. AO prepared the first draft of the manuscript. PP, LL, AM, and KS critically reviewed the manuscript. All authors reviewed the draft versions of the manuscript and approved the final version of the manuscript.

Authors' information

Not applicable
Ethics approval and consent to participate

This paper is a systematic scoping study relied strictly on the review of existing literature. Since there were no animal or human participants in this study, ethical approval is not required.

\section{Consent for publication}

Not applicable

Competing interests

The authors declare that they have no competing interests.

\section{Publisher's Note}

Springer Nature remains neutral with regard to jurisdictional claims in published maps and institutional affiliations.

\section{Author details}

${ }^{1}$ Department of Clinical Anatomy, School of Laboratory Medicine and Medical Sciences, University of KwaZulu-Natal, Durban, South Africa.

${ }^{2}$ Department of Plastic and Reconstructive Surgery, University of

KwaZulu-Natal, Durban, South Africa.

Received: 1 October 2018 Accepted: 14 January 2019

Published online: 04 February 2019

References

1. Zhou, et al. Diagnosis and classification of congenital craniofacial cleft deformities. Journal of craniofacial surgery. 2006;17.

2. Kawamoto HK. The kaleidoscopic world of rare craniofacial clefts: order out of chaos (Tessier classification). Clin Plast Surg. 1976;3:529-72.

3. WHO. Report of meetings on international collaborative research on craniofacial anomalies 2004. Available: www.who.int/genomics/publications/ CFA Completed text.pdf

4. WHO report on congenital Anomalies, September 2016. Available: http:// www.who.int/mediacentre/factsheets/fs370/en/

5. WHO. 2017. Sustainable Development Goals (SDGs) [Online]. Available: http://www.who.int/entity/sdg/en/

6. Chen PK-T, et al. Repair of Tessier no. 3 and no. 4 craniofacial clefts with facial unit and muscle repositioning by midface rotation advancement without Z-plasties. Plast Reconstr Surg. 2012;129(6):1337-44.

7. Morgan AL, et al. Anthropometrically-based surgical technique for Tessier 3 cleft reconstruction. J Craniofac Surg. 2016.

8. Mai T Pham, A.R., A scoping review of the use of theory in studies of knowledge translation. Can J Occup Ther. PMC, 2014.

9. Levac D, Colquhoun H, O'Brien KK. Scoping studies: advancing the methodology. Implement Sci. 2010;5(1):69.

10. Arksey H, O'malley I. Scoping studies: towards a methodological framework. international journal of social research methodology. 2005;8:19-32.

11. Dohoo IR, Martin SW, Stryhn H. Methods in epidermiologic research; 2012.

12. Pluye P, Robert E, Cargo M, Bartlett G, O'cathain A, Griffiths F, Boardman F, Gagnon MP, Rousseau MC. Proposal: a mixed methods appraisal tool for systematic mixed studies reviews; 2011.

13. An J-g, et al. Surgical treatment for incomplete Tessier no.3 craniofacial cleft: report of three cases and review of literature. Zhonghua Kou Qiang Yi Xue Za Zhi = Zhonghua Kouqiang Yixue Zazhi = Chinese Journal Of Stomatology. 2012;47(3):164-8.

14. Kale SM, Pakhmode VK. Bilateral Tessier no. 4 facial cleft with left eye anophthalmos: a case report. Journal of the Indian Society of Pedodontics and Preventive Dentistry. 2000;18(3):87-9.

15. Valladares Mendías JC, et al. Macrostomia secondary to transverse facial cleft of Tessier no.4. Cirugia Pediatrica: Organo Oficial De La Sociedad Espanola De Cirugia Pediatrica. 1998;11(1):2-4.

16. Murawski E. Rare facial fissures (Tessier fissure no. 3-9). Pediatr Pol. 1981; 56(6):671-5.

17. Schlenker JD, et al. Classification of oblique facial clefts with microphthalmia. Plast Reconstr Surg. 1979;63(5):680-8.

18. Resnick Jl, Kawamoto HK Jr. Rare craniofacial clefts: Tessier no. 4 clefts. Plast Reconstr Surg. 1990;85(6):843-9.

19. Bartlett SP. Discussion: repair of Tessier no. 3 and no. 4 craniofacial clefts with facial unit and muscle repositioning by midface rotation advancement without Z-plasties. Plast Reconstr Surg. 2012;129(6):1345-6. 
20. Cizmeci O, Kuvat SV. Tessier no. 3 incomplete cleft reconstruction with alar transposition and irregular z-plasty. Plastic surgery international. 2011;2011:596569.

21. Rahpeyma A, Khajehahmadi S. Treatment of a unilateral Tessier number 4 facial cleft in an adult: role of nasolabial V-Y advancement flap. Br J Oral Maxillofac Surg. 2015;53(1):99-101.

22. Valle AS, et al. Fisura facial nasoorbitaria, tessier número 3. Naso-orbital cleft, Tessier Number 3. 2011;54(6):23-8.

23. Aleman RM, Martinez MG. Tessier 3 cleft in a pre-Hispanic anthropomorphic figurine in El Salvador, Central America. The Cleft Palate-Craniofacial Journal: Official Publication Of The American Cleft Palate-Craniofacial Association. 2017:54(2):227-30.

24. Nor, A.M., et al. (2016). Staged reconstruction of a Tessier number 4 facial cleft: a case report Bahrain Medical Bulletin vol 38 Pg 110-111.

25. AbdollahiFakhim S, et al. A bilateral Tessier number 4 and 5 facial cleft and surgical strategy: a case report. Iranian journal of otorhinolaryngology. 2013; 25(73):259.

26. Aköz T, et al. Bilaterally involved Tessier no. 4 cleft: case report. The Cleft palate-craniofacial journal. 1996;33(3):252-4.

27. Allam KA, et al. The Tessier number 3 cleft: a report of 10 cases and review of literature. J Plast Reconstr Aesthet Surg. 2014:67(8):1055-62.

28. Alonso $\mathrm{N}$, et al. Tessier no. 4 facial cleft: evolution of surgical treatment in a large series of patients. Plast Reconstr Surg. 2008;122(5):1505-13.

29. Balaji SM. Single stage correction of bilateral tessier 4 cleft. Indian Journal Of Dental Research: Official Publication Of Indian Society For Dental Research. 2017;28(1):105-8.

30. Coruh A, Gunay GK. A surgical conundrum: Tessier number 4 cleft. The Cleft palate-craniofacial journal. 2005;42(1):102-6.

31. da Silva Freitas $R$, et al. Oral-nasal-ocular cleft: the greatest challenge among the rare clefts. J Craniofac Surg. 2010;21(2):390-5.

32. Darzi MA, Chowdri NA. Oblique facial clefts: a report of Tessier numbers 3 , 4, 5, and 9 clefts. The Cleft palate-craniofacial journal. 1993;30(4):414-5.

33. Madaree A, et al. A method of repairing the no. 3 facial cleft. Br J Plast Surg. 1992:45(1):44-6.

34. Giglio A, et al. Rotation and advancement flap of the cheek in the treatment of rare craniofacial clefts. J Craniofac Surg. 2008;19(5):1411-5.

35. Horoz U, et al. A new surgical approach for Tessier no. 4 facial cleft reconstruction: lip-rescue flap. J Craniofac Surg. 2016;27(8):2138-40.

36. Mishra RK, Purwar R. Formatting the surgical management of Tessier cleft types 3 and 4. Indian Journal of Plastic Surgery. 2009:42(S1):S174-83.

37. Reddy SG, et al. Options for the nasal repair of non-syndromic unilateral Tessier no. 2 and 3 facial clefts. Indian Journal of Plastic Surgery. 2014;47(3):340-5.

38. Sari A, et al. Early bone grafting in Tessier number 4 cleft: a case report. Journal of Craniofacial Surgery. 2003;14(3):406-10.

39. Wenbin Z, et al. Tessier 3 cleft with clinical anophthalmia: two case reports and a review of the literature. The Cleft Palate-Craniofacial Journal: Official Publication Of The American Cleft Palate-Craniofacial Association. 2007:44(1):102-5.

40. Wu D, et al. Severe bilateral Tessier 3 clefts in a Uighur girl: the significance and surgical repair. Journal of Cranio-Maxillo-facial surgery: official publication of the European association for Cranio-Maxillo-facial Surgery. 2013;41(7):598-602.

41. Xu Y, et al. Two rare cases of simultaneous Tessier number 3 cleft, contralateral cleft lip, and signs of amniotic band syndrome. J Craniomaxillofac Surg. 2016:44(6):672-5.

42. Sigler $\mathrm{MO}$, et al. A rare craniofacial cleft: numbers 7, 2, and 3 clefts accompanied by a single median lip pit. The Cleft Palate-Craniofacial Journal: Official Publication Of The American Cleft Palate-Craniofacial Association. 2004;41(3):327-31.

43. Uemura T, et al. Composite Z plasty for cicatricial ectropion of Tessier III cleft. J Craniofac Surg. 2004;15(1):51-3.

44. Tokioka K, et al. Two cases of Tessier no. 4 cleft with anophthalmia. The Cleft palate-craniofacial journal. 2005;42(4):448-52.

45. Spolyar JL, et al. Tessier no. 3 and no. 4 clefts: sequential treatment in infancy by pre-surgical orthopedic skeletal contraction, comprehensive reconstruction, and novel surgical lengthening of the ala base-canthal distance. Journal of Cranio-Maxillo-facial surgery: official publication of the European association for Cranio-Maxillo-facial Surgery. 2015;43(7):1261-8.

46. Rintala A, et al. Oblique facial clefts. Scand J Plast Reconstr Surg. 1980; 14(3):291-7.

47. Portier-Marret N, et al. Complete bilateral facial cleft (Tessier 4) with corneal staphyloma: a rare association. J Pediatr Surg. 2008;43(10):e15-8.
48. Mishima K, et al. Three cases of oblique facial cleft. J Cranio-Maxillofac Surg. 1996:24(6):372-7.

49. Maeda T, et al. Combination of Tessier clefts 3 and 4: case report of a rare anomaly with 12 years' follow-up. J Cranio-Maxillofac Surg. 2014;42(8):1985-9.

50. Longaker MT, et al. Reconstruction of Tessier no. 4 clefts revisited. Plast Reconstr Surg. 1997;99(6):1501-7.

51. Laure B, et al. Tessier number 4 bilateral orbito-facial cleft: a 26-year followup. J Craniomaxillofac Surg. 2010;38(4):245-7.

52. Gawrych $\mathrm{E}$, et al. Tessier type 3 oblique facial cleft with a contralateral complete cleft lip and palate. Int J Oral Maxillofac Surg. 2010;39(11):1133-6.

53. Bodin F, et al. Craniofacial cleft: a case of Tessier no. 3, 7 and 11 cleft. J Plast Reconstr Aesthet Surg. 2006;59(12):1388-90.

54. Sesenna E, et al. Tessier 3 cleft with bilateral anophthalmia: case report and surgical treatment. Journal of Cranio-Maxillo-facial surgery: official publication of the European association for Cranio-Maxillo-facial Surgery. 2012:40(8):690-3.

\section{Ready to submit your research? Choose BMC and benefit from:}

- fast, convenient online submission

- thorough peer review by experienced researchers in your field

- rapid publication on acceptance

- support for research data, including large and complex data types

- gold Open Access which fosters wider collaboration and increased citations

- maximum visibility for your research: over $100 \mathrm{M}$ website views per year

At $\mathrm{BMC}$, research is always in progress.

Learn more biomedcentral.com/submissions 